\title{
Arguable ICD placement in a sarcoidosis patient with extensive cardiac involvement
}

Hanna Jankowska ${ }^{1}$, Karolina Dorniak ${ }^{1}$, Marcin Hellmann ${ }^{1}$, Anna Dubaniewicz ${ }^{2}$, Maria Dudziak ${ }^{1}$

${ }^{1}$ Department of Noninvasive Cardiac Diagnostics, Medical University of Gdansk, Gdansk, Poland

${ }^{2}$ Department of Pulmonology, Medical University of Gdansk, Gdansk, Poland

Submitted: 11 August 2017

Accepted: 15 October 2017

Arch Med Sci 2020; 16 (3): 707-708

DOI: https://doi.org/10.5114/aoms.2019.86708

Copyright $@ 2019$ Termedia \& Banach

Sarcoidosis is a systemic inflammatory disease of unclear etiology, with non-caseating granuloma formation most typically in the lungs and pulmonary lymph nodes. However, many organs can be involved, including the skin, the eyes, the brain and the heart. Cardiac involvement is the major cause of mortality in sarcoidosis. Death may result from either arrhythmia, heart block or heart failure [1, 2].

The diagnosis and management of cardiac involvement can be challenging. A 42-year-old man with a 10-year history of stage 2 pulmonary sarcoidosis confirmed by scalene biopsy presented with new onset dyspnea following an 8-year post-steroid remission. Computed tomography (CT) scan revealed lung disease progression. Two months later he was admitted for symptoms of cerebellar stroke. No atherosclerotic lesions were found on carotid Doppler. However, enlarged left ventricle (LV) with moderately reduced left ventricular ejection fraction (LVEF) and apical as well as basal inferolateral akinesia were found on echocardiography. On top of standard treatment for $\mathrm{LV}$ dysfunction, prednisone $80 \mathrm{mg}$ daily was initiated for suspected cardiac involvement, based on 24-hour ECG monitoring (episodes of nsVT, multifocal ventricular ectopy), echocardiography (LVEF 40\%, LVEDD $62 \mathrm{~mm}$ ), and subsequent cardiac magnetic resonance (CMR) findings (LVEF $36 \%$, LVEDD $66 \mathrm{~mm}$, areas of patchy or focal transmural late gadolinium enhancement (LGE), suggestive of sarcoid lesions). Cardiac magnetic resonance findings also included extensive subendocardial involvement in the apex and mid-anterior as well as basal postero-lateral wall and transmural involvement of the basal inferior wall, which required differential diagnosis for coronary artery disease (CAD). However, no angiographically detectable atherosclerosis was found on subsequent coronary angiography. Standard 12-lead ECG was normal. Highlights from 24-hour ECG, echocardiography, CMR and coronary angiography are compiled in Figure 1.

At 1-year follow-up, LV parameters remained largely unchanged (LVEF $39 \%$, LVEDD $63 \mathrm{~mm}$ ). Additionally, regional LV function assessment with speckle tracking revealed markedly reduced global longitudinal strain (GLS) of $-12 \%$, with deformation abnormalities noted in the vast majority of LV segments. Both standard LV parameters and strain abnormalities were unchanged on echocardiography repeated 6 months later. Moreover, multiple episodes of nsVT were recorded on repeated 24-hour ECG. Followup CMR revealed no new foci of LGE, with slight improvement of regional and global LV function $(\mathrm{LVEF}=40 \%)$ and no signs of active inflammation.

Due to granuloma formation and scarring that may involve any cardiac chamber, sarcoidosis can manifest as atrial as well as ventricular arrhyth-

\author{
Corresponding author: \\ Karolina Dorniak PhD \\ Department of Noninvasive \\ Cardiac Diagnostics \\ Medical University of Gdansk \\ 17 Smoluchowskiego St \\ 80-214 Gdansk, Poland \\ Phone: +48 605856315 \\ E-mail: karolina.dorniak@ \\ gmail.com
}



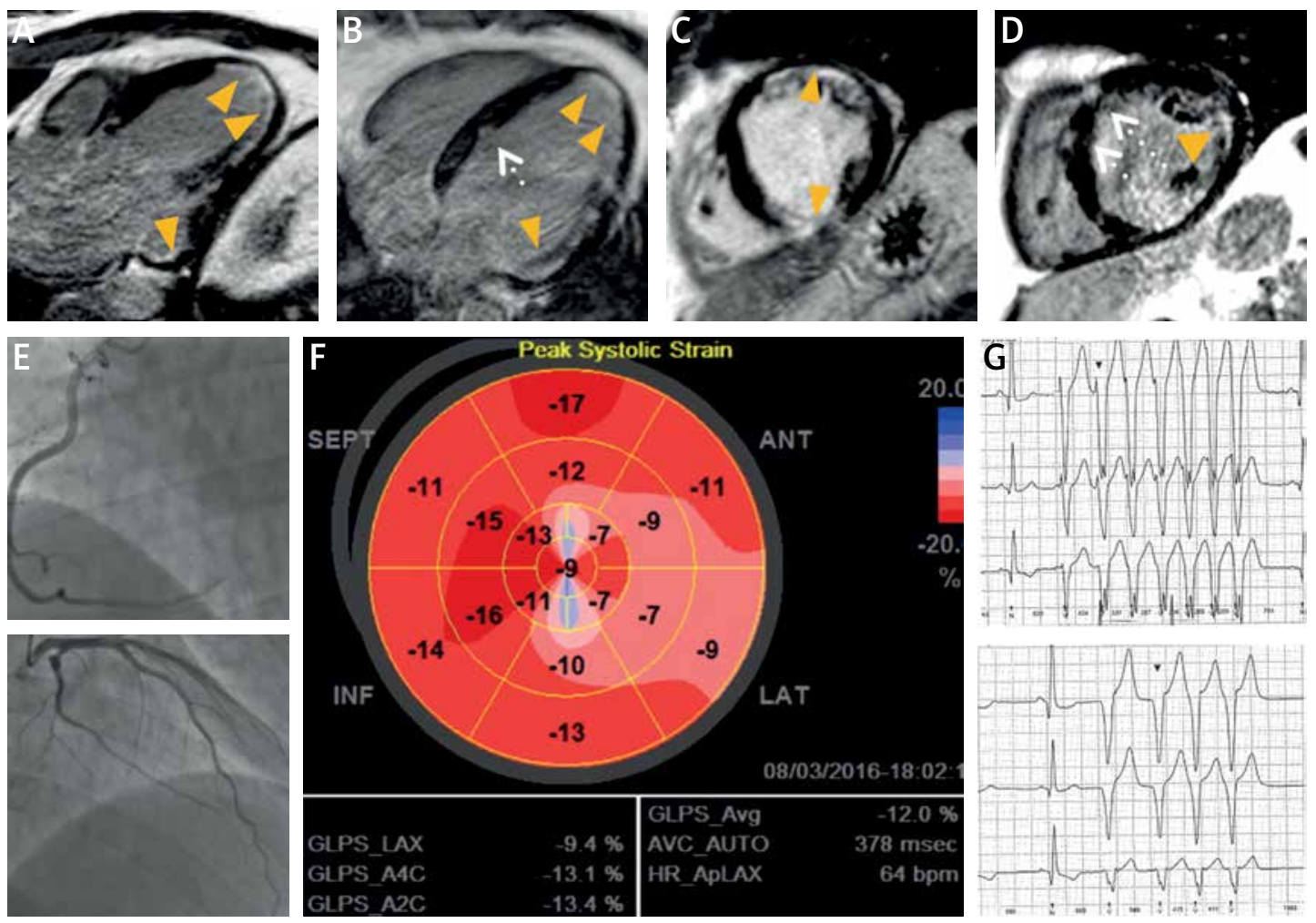

Figure 1. A, B - post-contrast inversion recovery long axis CMR images showing linear subendocardial (i.e. "CADtype") late gadolinium enhancement (LGE) in the apex and in the basal lateral and infero-lateral region (arrowheads) that mimic subendocardial infarctions; C, D - post-contrast inversion recovery short axis images showing subendocardial LGE in the mid-anterior, small transmural LGE in mid-inferior segment and basal lateral subendocardial LGE (arrowheads) that call for full CAD workup (On images B, D white dotted arrows point to small patchy areas of LGE that are typical for sarcoidosis and not for $(A D)$; $E$ - coronary angiography showing normal coronary arteries, the right (top) and the left (bottom) with no angiographically detectable atherosclerosis; $\mathbf{F}$ - global longitudinal LV strain showing significant impairment of myocardial deformation in all segments with the apex and entire lateral wall severely affected. G - Sample 24-hour ECG tracings showing recurrent nsVT

mias or heart block. The management of arrhythmia is challenging given the high recurrence rates and increased sudden cardiac death (SCD) risk. According to the 2014 Heart Rhythm Society recommendations [3], the patient was in class IIb for primary prevention with implantable cardioverterdefibrillator (ICD) placement. Given the extent of scarring, electrophysiology study (EPS), especially using advanced techniques such as ultra highdensity mapping [4], could have helped in the decision making. However, considering the aforementioned class Ilb recommendation (i.e. LVEF 36-49\%, despite optimal medical therapy and a period of immunosuppression, with risk assessment by CMR \pm EPS), and to avoid EPS-related delay, a pro-MRI ICD was placed based solely on follow-up findings, including lack of LV function improvement, extent of fibrotic lesions in the myocardium and recurring nsVTs despite adequate doses of a $\beta$-blocker and glucocorticosteroids. During 1-year follow-up after ICD placement, the patient remained in NYHA class II, no ICD intervention was recorded and the LV parameters were fairly stable.
In conclusion, this case of advanced cardiac sarcoidosis highlights the fact that the heart can be extensively damaged before any symptoms or complications of cardiac involvement occur. On the other hand, ICD placement is frequently not a straightforward decision in patients with sarcoidosis.

\section{Conflict of interest}

The authors declare no conflict of interest.

\section{References}

1. Judson MA. The clinical features of sarcoidosis: a comprehensive review. Clin Rev Allergy Immunol 2015; 49: 63-78.

2. Sekhri V, Sanal S, Delorenzo LJ, Aronow WS, Maguire GP. Cardiac sarcoidosis: a comprehensive review. Arch Med Sci 2011; 7: 546-554.

3. Birnie DH, Sauer WH, Bogun F, et al. HRS expert consensus statement on the diagnosis and management of arrhythmias associated with cardiac sarcoidosis. Heart Rhythm 2014; 11: 1305-23.

4. Nührich JM, Kaiser L, Akbulak RÖ, et al. Substrate characterization and catheter ablation in patients with scar-related ventricular tachycardia using ultra high-density 3-D mapping. J Cardiovasc Electrophysiol 2017; 28: 1058-67. 